\title{
Atividades Curriculares Pós-laborais em Medicina Geral e Familiar: Quanto Tempo a Mais?
}

\author{
Academic Activities of Family Doctors in Training: How Many Hours \\ Past the 40-hour Work Week?
}

Luís de Pinho Costa*, Elisabete Castro**, Sónia Marques Moreira***, Inês Valga Souto* ${ }^{* * * *}$, Catarina Pinto Nogueira ${ }^{* * * * *}$, Ana Cláudia Almeida******

\section{Resumo}

Introdução: A insatisfação dos internos de Medicina Geral e Familiar (MGF) com o tempo despendido em atividades curriculares não clínicas encontra-se documentada na literatura, contudo não existem dados sobre o tempo efetivamente despendido nessas atividades.

Objetivo: Caraterizar o tempo despendido pelos internos de MGF da Zona Norte em atividades curriculares no período pós-laboral e pesquisar a associação com variáveis sociodemográficas.

Métodos: Estudo observacional, transversal, descritivo e analítico; amostra de conveniência dos internos de MGF da Zona Norte, respeitando o tamanho amostral mínimo de 122 participantes. Foram enviados convites para participação, bem como questionário sociodemográfico e folha de registo do tempo despendido em 2 períodos de 7 dias consecutivos. Foram aplicados testes estatísticos para exploração de associações, tendo-se adotado um nível de significância de 0,05.

Resultados: A média de idades dos 122 participantes foi de 28 anos, sendo $84 \%$ do sexo feminino, 30\% casados ou em união de facto e 92\% sem descendentes a

\section{Abstract}

Introduction: The dissatisfaction of Portuguese General Practice (GP)/Family Medicine (FM) trainees with the time spent in non-clinical curricular activities is documented in the literature; however, there is no data on the time actually spent in these activities.

Objectives: To characterize the time spent by the Northern Portugal Family Medicine trainees in curricular activities carried out after working hours, and to investigate the association with sociodemographic variables.

Methods: Observational, cross-sectional, descriptive and analytical study; convenience sample of Northern Portugal GP/FM trainees, with a minimum sample size of 122 . Invitations for participation were sent, together with a sociodemographic questionnaire and a spreadsheet to record the time spent in 2 periods of 7 consecutive days. Statistical tests were used to explore associations, adopting a level of significance of 0.05 .

Results: The mean age of the 122 participants was 28 years, being $84 \%$ female, 30\% married or living together; $92 \%$ had no children. About $20 \%$ attended the 1 st year, $35 \%$ the 2nd year, 20\% the 3rd year and 25\% the fourth

\footnotetext{
* USF Amanhecer, ACeS Gondomar, ARS Norte

** USF Baguim, ACeS Gondomar, ARS Norte

*** USF Santa Maria, ACeS Gondomar, ARS Norte

**** USF Custóias, ACeS Matosinhos, ARS Norte

****** USF Fânzeres, ACeS Gondomar, ARS Norte

******* USF Tâmega, ACeS Baixo Tâmega, ARS Norte
} 
cargo. Cerca de $20 \%$ frequentavam o $1^{\circ}$ ano, $35 \%$ o $2^{\circ}$ ano, $20 \%$ o $3^{\circ}$ ano e $25 \%$ o $4^{\circ}$ ano de Internato. A mediana da carga horária despendida em semana típica foi de 18 horas. 0 estado civil demonstrou influenciar a carga horária semanal, sendo que os casados ou em união de facto despendem em média mais 4 horas e 30 minutos que os solteiros em atividades curriculares pós-laborais. A distribuição por tipo de atividade é liderada pela realização de trabalhos (4 horas), cursos (3 horas) e estudo (2 horas).

Discussão: As 40 horas do contrato de trabalho são a ponta do icebergue do tempo de trabalho efetivo dos internos de MGF. A confirmarem-se estes resultados, mediante estudos com uma metodologia mais robusta, importa realizar uma reflexão sobre os fatores causais e o seu impacto na valorização da trajetória de aprendizagem do interno.

Palavras-Chave: Internato e residência; estudo observacional; carga de trabalho; gestão do tempo.

\section{Introdução}

O Internato de Medicina Geral e Familiar (MGF) apresenta uma duração de quatro anos, com objetivo de habilitar o médico ao exercício tecnicamente diferenciado da especialidade. A avaliação individual exige o cumprimento de objetivos de desempenho e de conhecimento na área respetiva. ${ }^{1-5}$

A conclusão do Internato de MGF prevê a realização de uma avaliação final que inclui três provas, designadamente as provas de discussão curricular, teórica e prática. A Ordem dos Médicos homologou, a 21 de março de 2016, os critérios de valorização curricular relativos a estágios hospitalares, trabalhos científicos, cursos frequentados, programas doutorais de investigação clínica, contributos para a formação de outros profissionais, participação em organizações socioprofissionais, entre outras atividades. Adicionalmente, o interno deve, ao longo da sua formação, investir na atualização técnicocientífica que será testada em vários momentos do seu percurso formativo e nas provas referidas. ${ }^{1-7}$ Os critérios acima determinados são definidos para efeitos do presente estudo como "atividades curriculares".

Como definido no artigo $38^{\circ}$, Portaria $n^{\circ} 79 / 2018$, e no artigo $15 .^{\circ}$ do Decreto de Lei n 266-D/2012, o horário dos médicos internos é estabelecido e programado tendo em conta as atividades específicas do respetivo year of training. The median of the workload spent on a typical week was 18 hours. Marital status has been shown to influence weekly workload, with married or living together trainees spending an average of 4 hours and 30 minutes more than singles in after work hours curricular activities. The distribution by type of activity is led by the accomplishment of scientific/academic work (4 hours), courses (3 hours) and study time ( 2 hours).

Discussion: The 40 hours of the employment contract are the tip of the iceberg of the effective working time of Northern Portugal Family Medicine trainees. If confirmed by more robust studies, a deeper reflection will be needed on the causal factors and the impact on the valuation of the learning/training trajectory.

Keywords: internship and residency; observational study; workload; time management

programa de formação, sendo o período normal de trabalho de 40 horas semanais. Durante esse período, o tempo é despendido maioritariamente em atividades assistenciais, sendo possível que a carga horária seja ultrapassada com a realização de outras atividades de valorização curricular. ${ }^{1-9}$

\section{Objetivo principal}

- Determinar a carga horária semanal média despendida em atividades curriculares no período pós-laboral, pelos internos de MGF da Zona Norte

\section{Objetivos secundários}

- Determinar a carga horária semanal média despendida em atividades curriculares no período pós-laboral, em semana típica e por categoria de atividade curricular;

- Caracterizar a relação entre a carga horária e variáveis sociodemográficas - idade, sexo, estado civil, número de filhos, ano de Internato e Direção de Internato;

- Caracterizar a relação entre a carga horária e a estimativa realizada pelos internos. 


\section{Resultados expectáveis}

A formação do Médico de Família é um processo exigente que implica gestão do horário laboral e pós-laboral. Com base num inquérito publicado em 2014 que revelou insatisfação dos Internos com o tempo despendido em atividades não clínicas para efeitos curriculares, os investigadores levantam a hipótese que estas atividades ocupem um tempo considerável do período pós-laboral. ${ }^{10}$ Dada a necessidade de equilíbrio entre trabalho e vida privada postula-se que as variáveis sociodemográficas possam estar relacionadas com o tempo despendido; os autores tinham como hipótese que Internos solteiros e com menos descendentes despendessem mais tempo em atividades curriculares. Dado não existirem dados relativos ao tempo despendido pelos Internos de MGF nestas atividades, julga-se pertinente o seu estudo.

\section{Métodos}

Foi realizado um estudo observacional, descritivo com componente analítica. A população corresponde aos Internos de MGF da Zona Norte ( $N=895)$. Foi realizada amostragem de conveniência dos Internos com endereço eletrónico na Coordenação de Internato de MGF da Zona Norte (CIMGFZN). O tamanho amostral mínimo foi calculado em 122 participantes, com intervalo de confiança a 95\%, assumindo um desvio padrão de 6 horas e erro aceitável de 3 horas.

Após obtenção de parecer favorável da CIMGFZN, Comissão de Ética para a Saúde da Administração Regional de Saúde (ARS) do Norte e Comissão Nacional de Proteção de Dados (CNPD), procedeu-se à divulgação eletrónica do convite para participação.

As variáveis estudadas foram: sexo, idade, estado civil, número de descendentes, Direção de Internato e ano de Internato; estimativa da carga horária semanal média em atividades curriculares no período pós-laboral e carga horária semanal média despendida em atividades curriculares no período pós-laboral (total, parcelar por tipo de atividade e total em semana típica). A "semana típica" foi definida com base na correspondência entre o tempo objetivado e o tempo estimado pelo interno. Tendo em conta o reduzido número de respondentes que tinham filhos, a variável discreta "Número de descendentes" foi dicotomizada nas categorias "sem filhos" e "um ou mais filhos".
Entendeu-se por atividades curriculares aquelas que cumpriam pelo menos um dos critérios: 1) estavam previstas no programa do Internato de MGF (Diário da República, 1. ${ }^{a}$ série - N. ${ }^{\circ} 58$ - 24 de março de 2009); 2) constituíam elementos de valorização curricular pelos critérios homologados pela Ordem dos Médicos; 3) estavam definidas pela CIMGFZN ou pela respetiva Direção de Internato. Estas atividades contemplam o estudo, a elaboração de relatórios, a caracterização da comunidade, o estudo das famílias, a caracterização geral da lista de utentes, a análise das atividades assistenciais, as contribuições para a promoção institucional, a participação em cursos, a realização de trabalhos científicos, a formação de outros profissionais, os programas doutorais em investigação clínica e a participação em organizações socioprofissionais.

Entendeu-se por horário pós-laboral os períodos não contemplados no horário semanal de trabalho. A estimativa em horas e minutos (hh:mm) da carga horária semanal média em atividades curriculares no período pós-laboral teve em conta a totalidade do percurso formativo do Interno.

A variável de desfecho primária foi definida como a carga horária semanal média despendida em atividades curriculares no período pós-laboral, pelos Internos de MGF da Zona Norte. Como variáveis secundárias foram consideradas as cargas parcelares por atividade e a carga total numa semana típica. Para as cargas parcelares foram consideradas as categorias: "estudo"; "elaboração de relatórios", "promoção institucional”; "cursos/ pós-graduações"; "trabalhos científicos"; "formação de outros profissionais"; "participação em organizações socioprofissionais" e "outras atividades".

As variáveis de desfecho foram operacionalizadas através do cálculo da média de dois períodos de sete dias consecutivos (não interrompidos por férias ou comissões gratuitas de serviço), intervalados de, pelo menos, três semanas. A determinação da carga horária semanal foi efetuada com base no somatório do tempo (em horas e minutos - hh:mm) despendido nas atividades supramencionadas, em horário pós-laboral, em cada período de sete dias, mediante o reportado pelo participante. Foi disponibilizada uma folha de cálculo em formato .xls (Livro do Exce ${ }^{\circledR}$ 97-2003), elaborada pelos investigadores (vide Figura 1), para registo da categoria de atividade, respetiva hora de início e de fim. 
Figura 1. Folha de registo disponibilizada aos participantes do estudo.

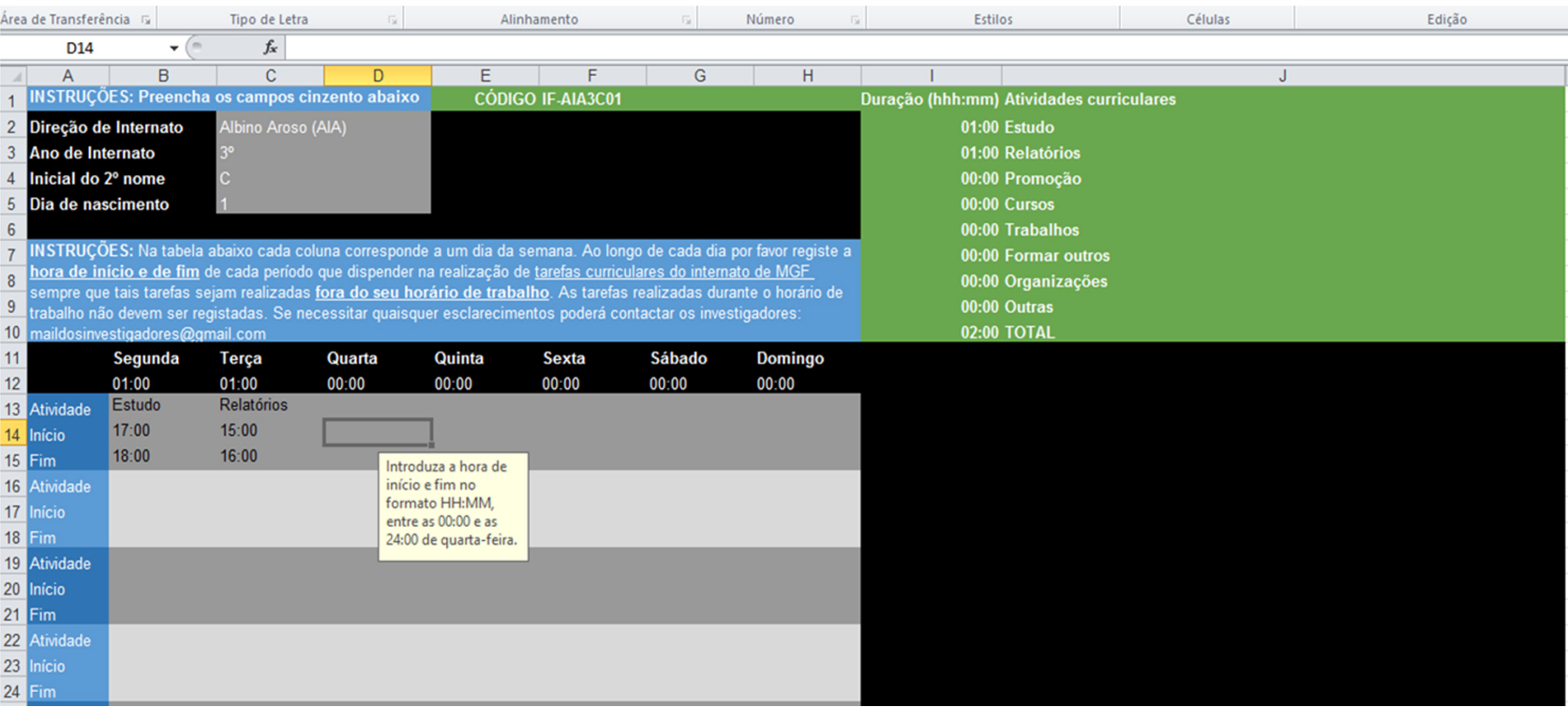

Na tipicidade dos períodos admitiram-se três respostas mutuamente exclusivas: "Foi uma semana típica", "Despendi menos horas que o habitual nas atividades curriculares em período pós-laboral" ou "Despendi mais horas que o habitual nas atividades curriculares em período pós-laboral".

A colheita dos dados sociodemográficos, da estimativa da carga horária semanal média em atividades curriculares no período pós-laboral e da data de início do registo das atividades curriculares pós-laborais (primeiro período) foi realizada com recurso a um questionário de autopreenchimento desenvolvido pelos investigadores; a hiperligação para este questionário foi disponibilizada no convite enviado. Neste constou também a senha solicitada no início do questionário, para evitar a participação de elementos externos à população alvo, dado o questionário estar alojado num local da internet de domínio público. Durante os períodos de registos das atividades curriculares pós-laborais foram enviados lembretes solicitando o preenchimento da folha de cálculo.

A colheita da carga horária semanal despendida nas atividades supramencionadas, em horário pós-laboral, em cada período de sete dias, foi realizada através de dois questionários de autopreenchimento desenvolvidos pelos investigadores, um para cada período.
Os questionários e a folha em Excel ${ }^{\circledR}$ para registo das atividades curriculares foram testadas e ajustados através de estudo piloto, em seis indivíduos com as mesmas características definidas para a amostra. Os três questionários foram construídos com recurso ao Google Forms ${ }^{\circledR}$, sendo que as respostas dos participantes geraram de forma automática as respetivas bases de dados. A correspondência entre os dados dos participantes nas três bases de dados foi assegurada pela existência de identificador único. Este identificador foi gerado automaticamente pela folha de cálculo disponibilizada para registo das atividades curriculares. Os resultados são apresentados com estimativas pontuais e intervalos de confiança a 95\%. A exploração de associações relevantes foi realizada com recurso a regressão linear simples, tendo-se adotado nível de significância de 0,05.

\section{Resultados}

Foram obtidas 122 respostas, 14\% da população. A distribuição por variáveis de interesse encontra-se resumida no Quadro 1. 
Quadro 1. Dados sociodemográficos e caracterização da amostra.

\begin{tabular}{|c|c|c|c|}
\hline \multirow[b]{2}{*}{ Categoria de dados } & \multirow[b]{2}{*}{ Variáveis } & \multicolumn{2}{|c|}{ Frequência } \\
\hline & & Absoluta (n) & Relativa (\%) \\
\hline \multirow{2}{*}{ Sexo } & Feminino & 102 & 84 \\
\hline & Masculino & 20 & 16 \\
\hline \multirow{4}{*}{ Estado civil } & Solteiro & 86 & 70 \\
\hline & Casado ou união de facto & 36 & 30 \\
\hline & Divorciado ou separado de facto & 0 & 0 \\
\hline & Viúvo & 0 & 0 \\
\hline \multirow{2}{*}{$\mathrm{N}^{\circ}$ de descendentes a cargo } & Nenhum & 112 & 92 \\
\hline & 1 ou mais & 10 & 8 \\
\hline \multirow{4}{*}{ Ano de Internato } & $1^{\circ}$ & 24 & 20 \\
\hline & $2^{\circ}$ & 43 & 35 \\
\hline & $3^{\circ}$ & 24 & 20 \\
\hline & $4^{\circ}$ & 31 & 25 \\
\hline \multirow{15}{*}{ Direção de Internato } & Abel Salazar & 7 & 5,7 \\
\hline & Albino Aroso & 15 & 12,3 \\
\hline & Camilo Castelo Branco & 7 & 5,7 \\
\hline & Corino de Andrade & 7 & 5,7 \\
\hline & Egas Moniz & 14 & 11,5 \\
\hline & Elísio de Moura & 3 & 2,5 \\
\hline & Emílio Peres & 5 & 4,1 \\
\hline & Jacinto Magalhães & 4 & 3,3 \\
\hline & José da Paz & 11 & 9,0 \\
\hline & Júlio Dinis & 10 & 8,2 \\
\hline & Miguel Torga & 5 & 4,1 \\
\hline & Nuno Grande & 10 & 8,2 \\
\hline & Ricardo Jorge & 7 & 5,7 \\
\hline & Santos Silva & 15 & 12,3 \\
\hline & Trindade Coelho & 2 & 1,6 \\
\hline
\end{tabular}

A mediana da estimativa da carga horária semanal despendida em atividades curriculares pós-laborais foi de 12 horas (percentil 25: 10 horas; percentil 75: 16 horas). Os períodos de resposta foram considerados “típicos" por 79\% dos Internos. A mediana do tempo despendido em atividades curriculares pós-laborais em semana típica foi de 18 horas. Relativamente à distribuição por categoria de atividade, destacam-se os trabalhos científicos, os cursos e o estudo (Quadro 2).

Quadro 2. Distribuição por categoria de atividade.

\begin{tabular}{l|c|c} 
Atividades & \multicolumn{1}{c}{$\begin{array}{c}\text { Mediana } \\
\text { (horas e minutos) }\end{array}$} & $\begin{array}{c}\text { P25 } \\
\text { (horas e minutos) }\end{array}$ \\
\hline Trabalhos científicos & $04: 22$ & $02: 00$ \\
\hline Cursos & $03: 00$ & $00: 00$ \\
\hline Estudo & $02: 30$ & $00: 15$ \\
\hline Relatórios & $01: 00$ & $00: 00$ \\
\hline Promoção Institucional & $00: 00$ & $00: 00$ \\
\hline Formação de outros profissionais & $00: 00$ & $00: 00$ \\
\hline Participação em organização socioprofissionais & $00: 00$ & $00: 00$ \\
\hline Outras atividades & $00: 00$ \\
\hline Total & $18: 00$ & $00: 00$ \\
\hline
\end{tabular}


Verificou-se que os Internos casados ou a viver em união de facto despenderam, em termos medianos, um número de horas semanal superior aos solteiros (20:15 versus 17:18 horas; $p<0.05)$. Nenhuma das outras variáveis mostrou significado estatístico (Quadro 3).

Quadro 3. Pesquisa de associação com variáveis demográficas.

\begin{tabular}{|c|c|c|c|}
\hline \multicolumn{2}{|l|}{ Variáveis } & $\begin{array}{c}\text { Mediana } \\
\text { (em horas e minutos) }\end{array}$ & \multirow{2}{*}{$\begin{array}{l}\text { Valor } p \\
0,823^{1}\end{array}$} \\
\hline Idade & & & \\
\hline \multirow{2}{*}{ Sexo } & Masculino & $15: 50$ & \multirow{2}{*}{$0,234^{2}$} \\
\hline & Feminino & $18: 45$ & \\
\hline \multirow{2}{*}{ Estado Civil } & Solteira (a) & $17: 18$ & \multirow{2}{*}{$0,049^{2}$} \\
\hline & Casada (o)/União de facto & $20: 15$ & \\
\hline \multirow{2}{*}{$N^{\circ}$ de descendentes } & Nenhum & $17: 30$ & \multirow{2}{*}{$0,066^{2}$} \\
\hline & 1 ou mais & $22: 55$ & \\
\hline \multirow{4}{*}{ Ano de Internato } & $1^{\circ}$ & $16: 25$ & $0,071^{3}$ \\
\hline & $2^{\circ}$ & 20:00 & \\
\hline & $3^{\circ}$ & $19: 15$ & \\
\hline & $4^{\circ}$ & $16: 08$ & \\
\hline Estimativa da carga horária semanal & - & $12: 00$ & $<0,001$ \\
\hline
\end{tabular}

Legenda: ${ }^{1}$ Coeficiente de correlação de Spearman; ${ }^{2}$ Mann-whitneyU; ${ }^{3}$ Kruskal- Wallis

Quando construído o modelo de regressão ANOVA, o facto de ser casado ou viver em união de facto aumenta em média 4 horas e 30 minutos à carga horária semanal média despendida em atividades curriculares em horário pós-laboral, com significado estatístico $(p=0.027)$. As outras variáveis demográficas não foram incluídas no modelo.

A estimativa da carga horária e a carga horária efetivamente despendida nas atividades curriculares em horário pós-laboral mostraram estar correlacionadas positivamente (coeficiente de correlação de Spearman de 0,398; $\mathrm{p}<0,0001$ ), ou seja, os Internos que estimaram maior número de horas foram aqueles que despenderam mais tempo efetivo.

As Direções de Internato com uma mediana de horas despendidas mais elevadas foram Miguel Torga, Trindade Coelho e Egas Moniz - vide Quadro 4. 
Quadro 4. Distribuição por Direção de Internato.

\begin{tabular}{|c|c|}
\hline Direção de Internato & Mediana (em horas e minutos) \\
\hline Miguel Torga - ACES Alto Tâmega e Barroso, Douro I e II & 23:45 \\
\hline Trindade Coelho - ULS Nordeste & 22:45 \\
\hline Egas Moniz - ACES Entre Douro e Vouga I e II & 22:22 \\
\hline Elísio de Moura - ACES Cávado I, Cávado II, Cávado III & 21:19 \\
\hline Emílio Peres - ACES Grande Porto VI & 20:05 \\
\hline Albino Aroso - ACES Grande Porto II & 20:00 \\
\hline José da Paz - ACES Tâmega II & 19:00 \\
\hline Abel Salazar - ULS (Unidade Local de Saúde) de Matosinhos & 18:00 \\
\hline Santos Silva - ACES Grande Porto VII e Grande Porto VIII & $18: 50$ \\
\hline Jacinto Magalhães - ACES Tâmega I, Tâmega III & $17: 00$ \\
\hline Júlio Dinis - ACES Grande Porto V & $16: 32$ \\
\hline Camilo Castelo Branco - ACES Guimarães/Vizela/Terras de Basto e Ave Famalicão & $16: 15$ \\
\hline Ricardo Jorge - ULS Alto Minho & $16: 15$ \\
\hline Nuno Grande - ACES Grande Porto I, Grande Porto III & 15:30 \\
\hline Corino de Andrade - ACES Grande Porto IV & $11: 30$ \\
\hline
\end{tabular}

\section{Discussão}

O objetivo do presente trabalho foi avaliar a carga horária semanal dos Internos de MGF em atividades curriculares no período pós-laboral, dado não existirem dados que o permitissem objetivar. A população do estudo correspondeu aos Internos da CIMGFZN. No entanto, dada a reduzida participação (14\% da população alvo), não foi possível realizar uma amostragem por quotas proporcionais aos tamanhos relativos das diferentes Direções de Internato. É reconhecida a dificuldade em obter a participação em trabalhos científicos, incluindo pares médicos, nos diferentes contextos, com destaque para a variação das populações e do desenho dos estudos. Nesse sentido foram implementadas as estratégias que se afiguraram aplicáveis de entre as descritas no corpo de evidência científica: o convite para o estudo foi enviado aos participantes pela CIMGFZN, tendo-se numa segunda fase, dois meses depois, solicitado o seu reenvio; o estudo foi publicitado em reuniões das Direções de Internato bem como em fóruns online de uso restrito pela população em estudo; foi solicitado aos participantes a divulgação do estudo aos seus pares; foram asseguradas e transmitidas as garantias de confidencialidade, evitadas questões de natureza sensível e minimizado/explicitado o tempo previsto para o preenchimento dos questionários; foi assumido o compromisso de ser disponibilizado o artigo aos participantes; foram feitos contactos de seguimento e enviados lembretes de forma a diminuir as perdas de seguimento, com recurso a mensagens personalizadas e comunicação clara. 0 facto destas estratégias se terem revelado insuficientes leva os investigadores a considerarem premente o estudo de estratégias específicas para esta população. Ainda assim, o uso de amostragem de conveniência surge como ponto negativo pelo possível viés de seleção introduzido. Com efeito, a mediana do tempo despendido em horário pós-laboral na realização de atividades curriculares foi superior ao estimado pelos Internos; tal resultado difere do obtido em estudos em outros contextos, os quais demonstram que a monitorização pelo indivíduo do tempo de trabalho poderá ser sobrevalorizada. ${ }^{8}$ Tal diferença poderá ser explicada por expectativas desajustadas da capacidade de trabalho e/ ou gestão inadequado do tempo despendido. Por outro lado, não foi possível excluir enviesamento pela não resposta, dado que os investigadores não tiveram acesso às características sociodemográficas dos não respondedores para comparação com as da população alvo. 
A monitorização de apenas duas semanas constitui limitação adicional, dado que, atendendo a que a carga de trabalho poderá ser mais acentuada em determinados períodos (por exemplo, em época de exame ou de congressos médicos de maior relevo curricular). Por essa razão, os autores propuseram-se estudar a tipicidade da semana. No entanto, dada a reduzida participação, não foi possível a comparação das semanas típicas e atípicas. Foi decidido não considerar os dados das respostas "Despendi menos ou mais horas que o habitual" na ulterior análise estatística, uma vez que poderiam enviesar os resultados. Apesar das instruções para os participantes terem sido claras, os autores levantam a hipótese de alguns Internos terem incluído as horas não assistenciais na estimativa das horas despendidas em atividades curriculares em horário pós-laboral, o que introduz um viés de medição. A utilização do registo biométrico, por um período mais longo, seria um valioso instrumento para ajustar as estimativas obtidas neste estudo. Embora se admita que as estimativas possam estar inflacionadas, os autores registam a tendência universal de todos os participantes para o dispêndio de tempo pessoal (pós-laboral) na realização de atividades de valorização curricular. Adicionalmente, no presente estudo não foi avaliado o tempo despendido em eventos de atualização técnico-científica, pois são em geral frequentados com pedido de comissão gratuita de serviço; no entanto, a participação nestes eventos é esporádica ao longo do ano, muito embora possa ter duração superior à da jornada de trabalho da correspondente comissão gratuita de serviço.

A maioria dos participantes era do sexo feminino, solteiro e não possuía descendentes. Mais surpreendentemente, verificou-se que os internos casados ou em união de facto despendem mais tempo em atividades curriculares pós-laborais. Os autores levantam como hipótese uma gestão mais eficiente do tempo pelos internos solteiros; outra hipótese prende-se com uma preferência pessoal de maior investimento curricular nos internos casados. Por um lado, a satisfação exclusiva das prioridades individuais (por oposição à necessidade de conjugar prioridades dos dois elementos de um casal, que poderão ser até competitivas entre si) e um menor número de fatores distratores ou interrupções poderão contribuir para a maior eficiência dos internos solteiros na gestão do seu tempo. Por outro lado, a partilha de responsabilidade domésticas nos casados e unidos de facto poderá facilitar um maior investimen- to curricular e proporcionar um melhor equilíbrio entre vida profissional e pessoal/familiar. Mais estudos serão necessários para confirmar estes resultados e para explicar a plausibilidade das hipóteses levantadas. Em todo o caso, de acordo com este estudo, ter uma vida familiar, casar ou ter filhos, não afeta negativamente o tempo despendido em atividades curriculares pós-laborais; estes resultados têm relevância não só para os internos, mas também para os orientadores e unidades funcionais que, ao tomar conhecimento deste facto, não deverão recear que os internos façam esse investimento na vida pessoal. As coordenações de internato, orientadores e unidades funcionais deverão promover o equilíbrio entre a vida profissional e pessoal, tratando igualmente internos solteiros e casados ou unidos de facto, com ou sem filhos.

A ausência de significância estatística para as restantes variáveis sociodemográficos poderá significar que não existe de facto relação com a variável de resultado, achado que vai de encontro à expectativa inicial dos investigadores. Não se pode no entanto descartar que tal associação não tenha sido detetada devido ao reduzido tamanho amostral.

A motivação pessoal para a realização de atividades curriculares, não estudada neste trabalho, poderá ser considerado um viés de participação tendo em conta que os internos mais motivados poderão ter sido aqueles que decidiram participar.

Os autores destacam que o tempo despendido em atividades curriculares em horário pós-laboral pode ser explicado não só pela crescente pressão curricular, mas também pelo desenvolvimento científico verificado nos últimos anos.

Também seria interessante relacionar o tempo despendido em atividades curriculares pós laborais e o tempo despendido em atividade clinica assistencial, uma vez que as variações deste último poderão condicionar a disponibilidade e motivação para as atividades curriculares.

Seria pertinente avaliar a satisfação dos internos quanto ao tempo despendido nas atividades referidas, de forma a compreender o impacto da carga horária na sua qualidade de vida. Seria igualmente interessante comparar a perceção dos médicos enquanto internos e mais tarde enquanto especialistas. 
A aplicação deste estudo a outras Coordenações de Internato poderá trazer um ponto de comparação entre as várias regiões do país e uma perspetiva mais global (nacional) sobre o Internato de MGF. Este estudo deverá ser tido como exploratório, permitindo obter uma estimativa grosseira de uma realidade até então desconhecida, mas que possibilita também identificar um conjunto de limitações que precisam ser ponderadas e resolvidas em futuras investigações nesta área. Caso se confirmem estes resultados em estudos com maior tamanho amostral e metodologia mais robusta, afigura-se necessária uma reflexão aprofundada sobre os fatores causais do número de horas "extraordinárias" e do seu real impacto na valorização da trajetória de aprendizagem do interno. Se as 40 horas do contrato de trabalho são a ponta do iceberg do tempo de trabalho efetivo dos Internos de MGF da Zona Norte, há que pensar a reformulação e aperfeiçoamento do Internato: que atividades curriculares trazem mais valor acrescentado para o interno? Há aplicabilidade prática, no futuro clínico, de todas as atividades curriculares que são exigidas? Quais são supérfluas ou requeridas em moldes exageradamente penosos ou excessivamente aprofundados? Seria preferível um modelo de avaliação que permitisse maior flexibilidade na escolha da tipologia e do número de atividades curriculares a realizar? Justifica-se uma extensão do Internato que permita redistribuir atividades, promova um melhor balanço entre vida profissional/pessoal e potencie uma maior satisfação dos internos?

\section{Conflito de Interesses}

Os autores declaram não ter conflito de interesses.

\section{Endereço para Correspondência}

Luís de Pinho Costa: luisdepinhocosta@gmail.com

Elisabete Castro: emacastro@gmail.com

Sónia Marques Moreira: soniamarquesmoreira@gmail.com Inês Valga Souto: ines.valga@gmail.com Catarina Pinto Nogueira: catarinapnogueira89@gmail.com Ana Cláudia Almeida: claudia.ac.almeida@gmail.com

\section{Referências Bibliográficas}

1. Programa de formação do Internato Médico da área profissional de especialização de Medicina Geral e Familiar. Portaria n³00 - 24 de março de 2009.

2. Regulamento do Internato Médico. Portaria n 251 - 24 de junho de 2011.

3. Coordenação do Internato Complementar da Zona Norte. Caderneta de Estágio 2014. Porto: CIMGFZN, 2014.

4. Coordenação do Internato Complementar da Zona Norte. Portefólio do Interno 2014. Porto: CIMGFZN, 2014.

5. Colégio de Medicina Geral e Familiar e Conselho Nacional Executivo da Ordem dos Médicos. Critérios de Avaliação Final do Internato Médico de Medicina Geral e Familiar. 2012.

6. EURACT Educational Agenda. Authorized Portuguese translation, guaranteed by Luís Filipe Gomes as Portugal Council Representative, free for academic purposes. Edição portuguesa da ADSO; julho 2006.

7. The European Society of General Practice/Family Medicine. The European Definition of General Practice/Family Medicine. WONCA Europe 2011 Edition.

8. Granja M, Ponte C. 0 que ocupa os médicos de família? Caracterização do trabalho médico para além da consulta. Rev Port Med Geral Fam 2011; 27: 388-96.

9. Granja M, Ponte C, Cavadas LF. What keeps family physicians busy in Portugal? A multicentre observational study of work other than direct patient contacts. BMJ Open 2014;4:1-11.

10. Azevedo A, Domingues B, Moura J, Santos L. Estão os Internos satisfeitos com o Internato de Medicina Geral e Familiar? Rev Port Med Geral Fam 2014 Fev; 30(1): 24-30. 